\title{
Evacuation gastrique du maïs broyé chez le porc
}

\author{
J.P. LAPLACE, Chantal KABORÉ, J.C. CUBER \\ avec la collaboration technique de Brigitte MOUROT et A. VAN HECKE \\ I.N.R.A., Laboratoire de Physiologie de la Nutrition \\ Centre de Recherches zootechniques, F 78350 Jouy-en-Josas
}

\begin{abstract}
Résumé
L'évacuation gastrique après ingestion de maïs grain broyé est étudiée chez 15 porcs de $60 \mathrm{~kg}$ par la méthode des abattages, en raison de propriétés particulières des contenus gastriques qui interdisent l'usage de la fistulation de l'estomac. Trois animaux sont sacrifiés à chaque temps postprandial $(30 \mathrm{mn}, 1,2,4$ et $7 \mathrm{~h})$ pour établir la cinétique d'évacuation (fig. 2) de la matière fraîche, de la matière sèche, de l'amidon et de l'azote. On relève en particulier une certaine dissociation de l'évacuation de la matière fraîche et de la matière sèche. Les quantités résiduelles sont comparées à celles enregistrées antérieurement pour de l'orge ou du blé broyés, soit par abattages au temps $2 \mathrm{~h}$ (fig. 3), soit par fistulation ( 5 temps de collecte). L'évacuation gastrique de la matière sèche est globalement plus lente après ingestion de maïs broyé qu'après ingestion d'orge ou de blé soumis à la même technologie de broyage.
\end{abstract}

Mots clés : Porc, maïs, évacuation gastrique, céréale, digestion.

\section{Introduction}

Il a été souligné dans un précédent travail (LAPLACE et al., 1985) le caractère relativement incomplet des connaissances relatives à la digestion de céréales telles que l'orge ou le blé. Cette carence est encore plus marquée dans le cas du maïs dont les effets sur les bilans nutritionnels, les performances de croissance et la composition corporelle du porc ont été étudiés par Henry (1968), et pour lequel SauER, STOTHERs \& Phillips (1977) ont précisé la digestibilité apparente des acides aminés. La digestion gastrique du maïs n'a guère été étudiée que par Holmes, BaYleY \& HoRneY (1974) selon le mode de conservation (maïs grain, sec ou ensilé). Enfin, si l'on connaît pour des régimes à base d'amidon de maïs purifié le rythme d'évacuation gastrique (Cuber, Laplace \& Villiers, 1980 ; Laplace, Pons \& Simon, 1983) et celui de l'apparition des sucres réducteurs dans la veine porte (RERAT et al., 1974; Rerat, Vaissade \& VAugelade, 1984), les informations homologues n'existent pas pour le maïs céréale entière. 
Aussi le présent travail a pour but d'étudier l'évacuation de l'estomac chez le porc, recevant exclusivement du maïs grain présenté sous forme broyée, et de situer ces résultats par rapport à ceux enregistrés pour d'autres céréales.

\section{Matériel et méthodes}

A la différence des études antérieures fondées sur l'usage de la fistulation gastrique (Cuber, Laplace \& Villiers, 1980 ; Laplace, Pons \& Simon, 1983 ; Laplace et al., 1984), y compris pour des céréales comme l'orge et le blé (LAPLACE et al., 1985), le présent travail a recours à la méthode dite des abattages en série. Ce choix résulte d'observations préliminaires qui ont mis en évidence l'impossibilité de réaliser une collecte totale des contenus gastriques résiduels par une fistule gastrique lors d'ingestion de maïs, quelle qu'en soit la finesse de broyage. Il a été noté parallèlement, au cours de ces essais préliminaires, le fait que la distribution exclusive de maïs broyé conduit dans la majorité des cas à un refus alimentaire total à très brève échéance, ce qui interdit d'accoutumer les porcs au régime avant l'essai.

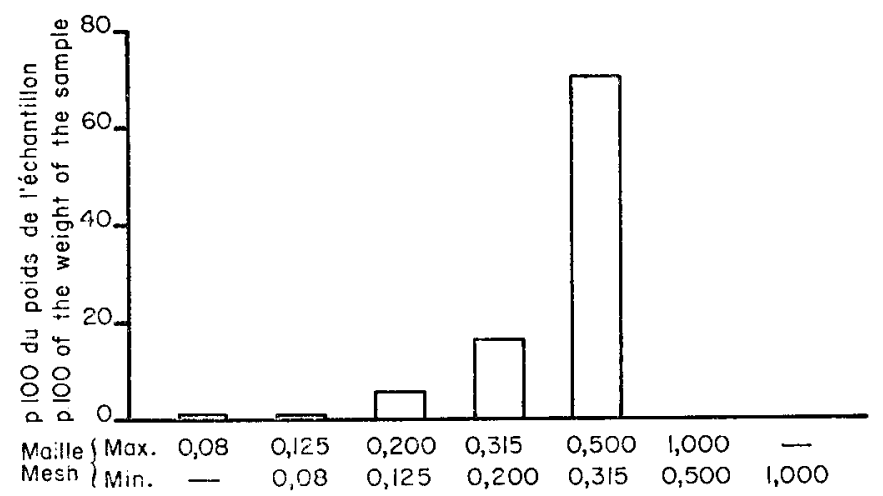

FIG. 1

Distribution moyenne des tailles des particules résultant du broyage du maïs grain entier au broyeur à marteaux équipé d'une grille de $1 \mathrm{~mm}$. Mesures obtenues par séparation sur des tamis à maille carrée de 1,0-0,5 - 0,315 - 0,2 - 0,125 et 0,08 mm.

Mean distribution of particle size of whole grain maize after hammer milling on a $1 \mathrm{~mm}$ screen. Measurements on multiple screens with square mesh of $1.0-0.5-0.315-0.2-0.125$ and $0.08 \mathrm{~mm}$.

Au total, 15 porcs mâles castrés de race Large White ont été utilisés. Leur poids vif moyen lors de l'abattage est de $60,3 \pm 1,5 \mathrm{~kg}$. Le maîs grain dont l'évacuation gastrique est étudiée a été broyé au broyeur à marteaux (Gondard, TB 12) équipé d'une grille de $1 \mathrm{~mm}$. La granulométrie du produit obtenu a été déterminée par tamisage durant $5 \mathrm{mn}$ à l'aide d'un appareil «Tamisor» équipé de six tamis à mailles carrées $(1,0-0,5-0,315-0,2-0,125-0,08 \mathrm{~mm})$. La distribution des tailles de particules est décrite par la figure 1. Ce maïs broyé est distribué lors des épreuves 
après addition d'un complément minéral et vitaminique dont la composition a été décrite par Henry \& RERAT (1966). L'aliment final comporte 96 p. 100 de maïs broyé et 4 p. 100 de ce complément (HENRY, 1968). La composition chimique du mélange est la suivante (en p. 100 de la matière sèche) : amidon, 66,5; azote, 1,2 ; cellulose brute (Weende), 2,0; résidu neutre, 12,0; résidu acide, 2,5 ; lignine, 0,2.

Les porcs ont été initialement hébergés en cage et alimentés en deux repas quotidiens à 9 et $16 \mathrm{~h}$, au cours des jours précédant l'abattage, avec l'aliment amidon de maïs purifié-farine de poisson antérieurement utilisé (LAPlace, Pons \& Simon, 1983). Le repas du soir est supprimé la veille de l'essai pour assurer la vacuité de l'estomac avant ingestion du repas d'épreuve. Au jour d'abattage les porcs reçoivent le matin à $9 \mathrm{~h}$, et donc sans accoutumance, l'aliment maïs broyé. Le repas d'épreuve représente $2500 \mathrm{~g}$ de matière fraîche (dont 1,51 d'eau de dilution) et $886 \mathrm{~g}$ de matière sèche dont $589 \mathrm{~g}$ d'amidon et $10,5 \mathrm{~g}$ d'azote. Les porcs ont été affectés au hasard à chacun des 5 temps postprandiaux choisis $(30 \mathrm{mn}, 1 \mathrm{~h}, 2 \mathrm{~h}, 4 \mathrm{~h}, 7 \mathrm{~h})$, à raison de 3 animaux dans chaque cas.

Environ 5 minutes avant l'heure fixée pour l'abattage, l'animal est anesthésié à l'halothane. On pratique une laparotomie permettant de procéder à l'heure choisie au clampage du pylore et du cardia. L'estomac est prélevé rapidement par section de l'œsophage abdominal, du duodénum proximal et de l'ensemble des mésos. L'animal est sacrifié cependant que l'estomac est essuyé avant ouverture. On procède alors à la récupération de la totalité du contenu gastrique résiduel à la faveur d'une incision de la grande courbure.

Les contenus gastriques recueillis sont pesés en l'état, homogénéisés vigoureusement et échantillonnés. Un premier échantillon d'environ $30 \mathrm{~g}$ est utilisé pour la détermination de la teneur en matière sèche (M.S.) par passage à l'étuve ( $24 \mathrm{~h}$ à $104^{\circ} \mathrm{C}$ ). Un second échantillon de même importance est prélevé puis lyophilisé en vue de la détermination de l'azote total par la méthode Kjeldhal et de l'amidon selon Thivend, Mercier \& Guilbot (1965). On peut ainsi calculer les quantités totales de matière sèche, amidon et azote, résiduelles dans l'estomac aux divers temps de collecte.

\section{Résultats}

Les quantités résiduelles de matière fraîche, matière sèche, amidon et azote, collectées aux 5 temps postprandiaux d'abattage (fig. 2) permettent de reconstituer une « cinétique " d'évacuation gastrique du maïs broyé. L'évacuation s'avère relativement rapide dans les premiers instants après le repas, puisque 20 à 25 p. 100 des quantités ingérées de matière sèche, amidon et azote ne sont plus retrouvées dans l'estomac au temps $30 \mathrm{mn}$. Cette évacuation rapide initiale est suivie d'une période d'évacuation beaucoup plus lente entre $30 \mathrm{mn}$ et $2 \mathrm{~h}$ dans le cas de la matière fraîche et à un moinde degré de l'azote, et entre $30 \mathrm{mn}$ et $1 \mathrm{~h}$ pour la matière sèche et l'amidon. Au-delà de cette phase l'évacuation se poursuit à nouveau à un rythme relativement rapide. 


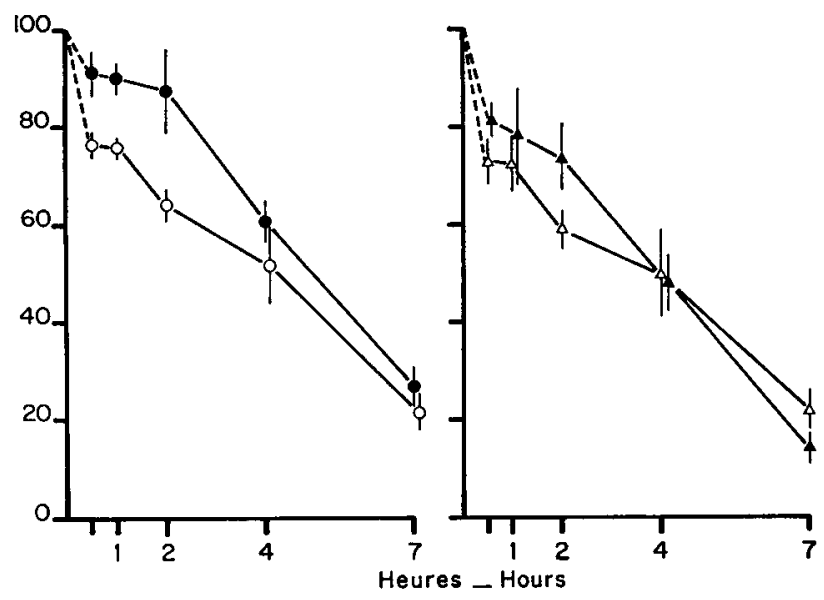

FIG. 2

Quantités résiduelles moyennes (et écarts types des moyennes) de matière fraîche (๑), matière sèche $(O)$, amidon $(\Delta)$ et azote $(\Delta)$ collectées dans l'estomac de porcs sacrifiés à divers temps après ingestion d'un aliment à base $(96 \mathrm{p} .100)$ de maïs broyé.

Valeurs exprimées en p. 100 des quantités ingérées correspondantes. $n=3$ porcs dans chaque cas.

Mean ( \pm SEM) residual quantities of wet digesta $(\bullet)$, dry matter $(\bigcirc)$, starch $(\triangle)$ and nitrogen (A) collected from the stomach of pigs slaughtered at different times after intake of a ground maize based (96 per cent) diet. Values as per cent of the respective ingested quantities. $n=3$ pigs in each case.

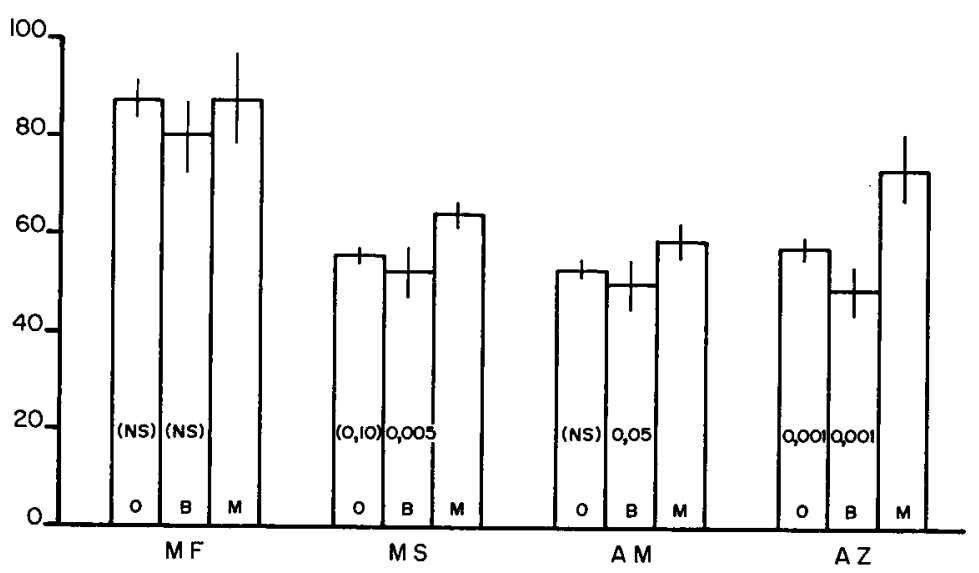

FIG. 3

Quantités résiduelles moyennes (et écarts types des moyennes) de matière fraîche $(M F)$, matière sèche $(M S)$, amidon $(A M)$ et azote $(A Z)$ collectées dans l'estomac de porcs sacrifiés $2 h$ après ingestion d'un aliment à base d'orge $(O, n=6)$, de blé $(B, n=6)$ ou de maïs $(M, n=3)$. Valeurs exprimées en p. 100 des quantités ingérées correspondantes.

Mean ( \pm SEM) residual quantities of wet digesta $(M F)$, dry matter $(M S)$, starch (AM) and nitrogen $(A Z)$ collected from the stomach of pigs slaughtered 2 hours after feeding $a$ diet based on either barley $(O, n=6)$, wheat $(B, n=6)$ or maize $(M, n=3)$. $V$ alues as percent of the respective ingested quantities. 
La comparaison des quantités résiduelles recueillies chez les 3 porcs sacrifiés $2 \mathrm{~h}$ après le repas à base de maïs à celles enregistrées chez 6 porcs sacrifiés également $2 \mathrm{~h}$ après ingestion d'aliments à base d'orge ou de blé broyés (LAPLACE et al., 1985) permet un bilan comparatif du résultat de l'évacuation gastrique au terme des 2 premières heures postprandiales (fig. 3 ). Les quantités résiduelles de matière sèche, amidon et azote sont significativement plus élevées pour le maïs que pour le blé ; seules celles d'azote sont significativement plus importantes pour le maïs que pour l'orge.

\section{Discussion}

Le cas du maïs appelle quelques observations d'ordre méthodologique. Il est en effet remarquable que la collecte des contenus gastriques résiduels par une fistule gastrique permanente ne soit pas possible lors d'ingestion de maïs broyé alors qu'elle est assurée de façon complète pour de l'orge ou du blé broyés (LAPLAcE et al., 1985). Cette particularité, qui impose l'usage de la technique des abattages dans le cas du maïs, ne permet évidemment pas du tout la même approche statistique de l'évacuation que celle qui résulte d'une étude chez des porcs fistulés. Ceci étant, il est évident que, pour une même technologie de broyage sur grille de $1 \mathrm{~mm}$, la distribution des tailles des particules enregistrée pour le maïs est très différente de celle observée pour l'orge et le blé (LAPLACE et al., 1985). La proportion relative de "grosses » particules $(>0,315 \mathrm{~mm})$ est en effet d'environ 70 p. 100 dans le cas du maïs contre 47 à 48 p. 100 pour l'orge et le blé. Mais cette caractéristique de granulométrie n'est pas nécessairement seule responsable de la difficulté d'écoulement des contenus gastriques : même après un rebroyage du maïs sur grille de $0,5 \mathrm{~mm}$ (testé lors des essais préliminaires), la collecte ne peut être assurée par une fistule chronique.

Dans tous les cas, l'ingestion de maïs broyé produit dans l'estomac des porcs une masse extrêmement épaisse. Du reste, le mélange in vitro d'eau et d'aliment, dans les mêmes proportions que celles mises en cuvre pour la présentation aux porcs, permet d'observer la différence d'aspect du mélange après 2 heures selon la céréale : L'orge fournit une bouillie homogène relativement fluide; par contre la suspension de particules obtenue pour le blé et le maïs fait l'objet d'une décantation. Mais le dépôt est très labile dans le cas du blé avec un surnageant important, alors que pour le maïs, le dépôt est extrêmement compact et surmonté d'une faible quantité de surnageant. A noter aussi que pour des poids identiques de farine et d'eau, le volume résultant est différent selon la céréale. La question est donc posée, d'une part de la nature du processus physique qui conduit dans le cas du maïs à la formation d'un véritable «pâté " intragastrique, et d'autre part des conséquences particulières de ce phénomène à l'égard du déroulement de l'évacuation gastrique.

En toute rigueur, la différence de méthodologie mise en œuvre pour décrire ici la cinétique d'évacuation du maïs, ou de l'orge et du blé antérieurement (LAPLACE et al., 1985), jointe à d'autres différences (non accoutumance au régime et jeûne la veille au soir, dans le cas du maïs), interdit une véritable comparaison de la cinétique reconstituée pour le maïs (fig. 2) avec les cinétiques établies pour l'orge et le blé chez des porcs fistulés (LAPLACE et al., 1985). On devrait alors se borner à la comparaison (fig. 3) des bilans d'évacuation $2 \mathrm{~h}$ après le repas, réalisés pour les 3 céréales 
par abattage. Ceux-ci témoignent du fait que pour une même évacuation apparente de matière fraîche, celle de la matière sèche et de l'azote est plus faible pour le maïs que pour l'orge et le blé. Le cas de l'amidon est moins net et de surcroît la technique de dosage utilisée n'est pas la même dans les deux expériences. Sous les réserves exprimées plus haut, si l'on accepte comme une approximation plausible la comparaison des cinétiques au cours des 7 heures postprandiales, on peut aller au-delà de ce qui ressort des bilans lors d'abattage à $2 \mathrm{~h}$. Les quantités résiduelles de matière fraîche après ingestion de maïs s'écartent fort peu, en plus ou en moins, de celles recueillies pour l'orge et le blé, au moins jusqu'à la quatrième heure postprandiale. Cependant, à la décroissance très faible des quantités résiduelles dans le cas du maïs avant 2 h (équivalant à 3,5 p. 100 de l'ingéré, contre 11,0 ou 9,5 p. 100 dans le cas de l'orge ou du blé respectivement), succède une forte évacuation de matière fraîche entre 2 et 7 h (équivalant à 60,4 p. 100 de l'ingéré contre 42,9 ou 39,1 p. 100 pour l'orge et le blé respectivement). Sur la base du bilan d'évacuation après $7 \mathrm{~h}$, la matière fraîche semblerait donc évacuée plus rapidement dans le cas du maïs. Par contre les quantités résiduelles de matière sèche sont toujours les plus élevées pour le maïs qui, sur cette base, est donc évacué plus lentement que l'orge et le blé. La différence d'ingéré sec pour un même ingéré frais, soit 3 p. 100 de plus pour le maïs que pour l'orge, ne peut expliquer l'excédent de résidu sec gastrique à $4 \mathrm{~h}$ dans le cas du maïs par rapport à l'orge, l'écart équivalant à 15,2 p. 100 de l'ingéré sec.

Ce phénomène quelque peu paradoxal enregistré dans le cas du maïs par rapport à l'orge ou au blé, avec évacuation de quantités plus faibles de matière sèche, pour des quantités analogues puis plus grandes de matière fraîche, pourrait relever de plusieurs causes. On pourrait suspecter un niveau très différent de volume des sécrétions salivaire et gastrique selon la céréale ingérée, conduisant dans le cas du maïs à l'addition d'une quantité beaucoup plus importante d'eau secrétoire que dans le cas de l'orge ou du blé. Toutefois l'ampleur des distorsions enregistrées entre l'évacuation de la matière fraîche et celle de la matière sèche, pour la période 4 à $7 \mathrm{~h}$ après le repas, fait qu'un tel phénomène secrétoire ne saurait à lui seul rendre compte de la différence. On pourrait ainsi envisager que le maïs fixe une quantité d'eau plus importante, immobilisant à la fois l'eau de dilution de l'aliment et l'eau secrétoire ajoutée jusqu'à atteindre un certain seuil d'imbibition. Une telle fixation d'eau expliquerait qu'à l'évacuation de quantités relativement plus faibles de matière sèche corresponde celle de quantités analogues de matière fraîche, toujours relativement à l'orge ou au blé, jusqu'à $4 \mathrm{~h}$ après le repas. Par la suite, le seuil d'imbibition maximum du maïs étant atteint, l'état physique de la masse intragastrique autoriserait une évacuation rapide, d'autant plus que le volume résiduel intragastrique encore important implique une forte stimulation. Cette seconde phase pourrait alors répondre de l'accélération considérable de l'évacuation de la matière fraîche effectivement constatée à 4 et $7 \mathrm{~h}$ après le repas. Enfin on note que la teneur en matière sèche des contenus gastriques décroît régulièrement, de façon quasi linéaire, de 29 ou 30 p. 100 à $30 \mathrm{mn}$ à 17 ou 13 p. 100 à $7 \mathrm{~h}$ respectivement pour l'orge et le blé, alors que dans le même temps elle reste à peu près stationnaire pour le maïs $(29,5 \cdot 30,3-26,3$ $31,4-28,9$ p. 100 respectivement à $30 \mathrm{mn}, 1 \mathrm{~h}, 2 \mathrm{~h}, 4$ et 7 heures). Ceci revient à dire qu'au cours du temps les contenus résiduels sont de plus en plus "dilués » dans le cas de l'orge et du blé alors qu'ils restent toujours aussi « épais » dans le cas du maïs. Aussi, l'évacuation plus rapide de la matière fraîche entre 4 et $7 \mathrm{~h}$ pour le maïs entraîne-t-elle relativement plus de matière sèche que pour les autres céréales, ce qui pourrait rendre compte du rapprochement des bilans d'évacuation de la matière sèche à $7 \mathrm{~h}$. 
En conclusion, le maïs ingéré sous forme broyée tend à former dans l'estomac du porc une masse pâteuse aux caractéristiques physico-chimiques telles qu'on assiste, au moins durant les premières heures postprandiales, à une dissociation apparente de l'évacuation de la matière fraîche et de la matière sèche. La teneur en matière sèche des contenus gastriques reste stable dans le cas du maïs, alors qu'elle diminue de moitié en $7 \mathrm{~h}$ pour de l'orge ou du blé. Au total, la matière sèche d'un régime constitué à 96 p. 100 de maïs broyé est évacuée plus lentement que celle de régimes homologues à base d'orge ou de blé.

\section{Summary}

Gastric emptying of ground maize in the pig

Gastric emptying of ground maize grain was studied in 15 pigs of $60 \mathrm{~kg}$ live weight using the slaughtering method because the stomach fistulation technique cannot be used due to the particular characteristics of gastric contents.

Three animals were slaughtered at each post prandial time (30 min, 1, 24 and 7 h) to determine the kinetics of emptying (fig. 2) of wet digesta, dry matter, starch and nitrogen. Gastric emptying of wet digesta seemed to be dissociated from that of dry matter. Residual quantities were compared to those previously obtained with ground barley and wheat either in pigs slaughtered 2 hours after feeding, or in fistulated pigs ( 5 collection periods).

Gastric emptying of dry matter was slower after ground maize intake than after intake of barley and wheat subjected to the same grinding technique.

Key words : Gastric emptying, digestion, pig, maize, cereals.

Reçu en octobre 1981.

Accepté en juin 1985.

\section{Références bibliographiques}

Cuber J.C., Laplace J.P., Villiers P.A., 1970. Fistulation de l'estomac et contenus gastriques résiduels après ingestion d'un régime semi-purifié à base d'amidon de maïs chez le porc. Reprod. Nutr. Dévelop., 20, 1161-1172.

Henry Y., 1968. Utilisation comparée des céréales comme seuls aliments du porc pendant la période de finition. Ann. Zootech., 17, 183-197.

Henry Y., Rerat A., 1966. Utilisation des pommes de terre déshydratées et fraîches dans l'alimentation du porc en croissance en comparaison avec l'orge. Ann. Zootech., 15, 231-251.

Holmes J.H.G., BAYley H.S., HoRney F.D., 1974. Digestion of dry and high-moisture maize diets in the stomach of the pig. Br. J. Nutr., 32, 639-646.

Laplace J.P., Pons O., Cuber J.C., Kaboré C., 1984. Evacuation gastrique et composition en acides aminés des protéines du régime chez le porc. Ann. Zootech., 33, 59-72.

Laplace J.P., Pons O., Cuber J.C., Kaboré C., 1985. Evacuation gastrique de l'orge et du blé chez le porc. Ann. Zootech., 34, 265-282.

Laplace J.P., Pons O., Simon P., 1983. Révision des effets de l'amidon et des protéines du régime sur l'évacuation gastrique chez le porc. Ann. Zootech., 32, 507-524. 
Rerat A., Aumaitre A., Vaissade P., Vaugelade P., 1974. Mesure de l'absorption des glucides au cours de la digestion de l'amidon de maïs chez le porc. C.R. Acad. Sci., 279 D, 831-834.

Rerat A., Vaissade P., Vaugelade P., 1984. Absorption kinetics of some carbohydrates in conscious pigs. 2) Quantitative aspects. Br. J. Nutr., 51, 517-529.

Sauer W.C., Stothers S.C., Phillips G.D., 1977. Apparent availabilities of amino acids in corn, wheat, and barley for growing pigs. Can. J. anim. Sci., 57, 585-597.

Thivend P., Mercier Ch., Guilbot A., 1965. Dosage de l'amidon dans les milieux complexes. Ann. Biol. anim. Biochim. Biophys., 5, 513-526. 